\title{
Adaptação da Escala de Estratégias de Coping na adolescência numa amostra Portuguesa
}

\author{
Paulo César Azevedo Dias \\ Universidade Católica Portuguesa \\ Jose A. Garcia del Castillo Rodriguez \\ Universidade Miguel Hernández de Elche \\ Carmen López-Sánchez \\ Universidade de Alicante
}

\section{Resumo}

\begin{abstract}
A adolescência é uma fase desenvolvimental marcada por desafios. No sentido de conhecer melhor as estratégias que os adolescentes utilizam para lidar com o stress, pretendeu-se com este estudo apresentar as propriedades psicométricas da Escala de Estratégias de Coping na Adolescência (Burnett \& Fanshawe, 1997), testada junto de uma amostra de adolescentes portugueses, explorando diferenças em função do género e da idade. Os resultados permitiram encontrar uma estrutura factorial válida e com consistência interna genericamente adequada. Os dados permitem perceber diferenças nas estratégias de coping, com as moças a procurar mais apoio dos outros e melhoria pessoal, enquanto os rapazes utilizam mais frequentemente drogas ou o humor. Em função da idade verificouse uma relação positiva com o uso de substâncias e diminuição da procura de apoio da família e de profissionais. Os dados são discutidos à luz da literatura e implicações para a prática são apresentadas.
\end{abstract}

Palavras-chave: adolescência; coping; psicometria.

\begin{abstract}
Adaptation of the Adolescent Coping Strategies Scale in a Portuguese sample. Adolescence is a developmental period marked by challenges. In order to better understand the strategies that adolescents use to cope with stress, it is intended with this study to present the psychometric properties of the Adolescent Coping Strategies Scale (Fanshawe \& Burnett, 1997), tested with a sample of Portuguese adolescents, exploring differences by gender and age. The results allowed to find a valid and generally adequate internal consistency factor structure. The data allow to discern differences in coping strategies, with the girls to seek more support from others and personal improvement, while boys more often use of drugs or humor. We found a positive relationship between age and substance use and decreased demand for support from family and professionals. The data are discussed according to literature and implications for practice are presented.
\end{abstract}

Keywords: adolescence; coping; psychometrics.

\section{Resumen}

Adaptación de la Escala de Estrategias de Afrontamiento en la Adolescencia en una muestra Portuguesa. La adolescencia es un periodo de desarrollo marcado por los desafíos. En el presente estudio se presentan las propiedades psicométricas de la Escala de Estrategias de Afrontamiento en la Adolescencia (Fanshawe y Burnett, 1997) con el fin de comprender mejor las estrategias que utilizan los adolescentes para hacer frente al estrés. El instrumento ha sido probado en una muestra de adolescentes portugueses, explorando las diferencias por sexo y edad. Los resultados permitieron encontrar una estructura factorial con una consistencia interna válida y adecuada. Los datos permiten discernir las diferencias en las estrategias de supervivencia, donde las chicas tienden a buscar más apoyo de los demás y la mejora personal, mientras que los chicos emplean más a menudo el consumo de drogas o el demanda del humor. Dependiendo de la edad se verifica una relación con el consumo de sustancias y la disminución de la demanda de apoyo familiar y profesional. Los datos se analizan en base a la literatura y se presentan las implicaciones para la práctica.

Palabras clave: adolescencia; afrontamiento; psicometría. 
A adolescência é uma fase desenvolvimental marcada por desafios importantes a diversos níveis, desde os desafios escolares e de carreira, à relação com a família ou na rede de pares, até às próprias alterações hormonais, cognitivas e cerebrais (e.g.: Casey et al., 2010; Gestsdottir \& Lerner, 2008; Zimmer-Gembeck \& Skinner, 2008). Embora a literatura permita perceber que a maioria dos adolescentes é capaz de lidar eficazmente com estes desafios (Kenny, Dooley, \& Fitzgerald, 2013), cerca de um terço experimentam elevados níveis de stress (e.g.: Arbour-Nicitopouos, Faulkner, \& Irving, 2012; Schraml, Perski, Grossi, \& Simonsson-Sarnecki, 2011). Como referem em revisão Skinner e Zimmer-Gembeck (2007), esta reação tem o potencial de moldar as relações sociais, a sua fisiologia e o percurso desenvolvimental dos adolescentes, pelo que lhe importa perceber os agentes stressores e, especialmente, como os adolescentes lidam com estas situações, que estratégias de coping utilizam. Conhecendo estas estratégias, e intervindo adequadamente, podemos potenciar o ajustamento individual, a saúde e o bem-estar nesta faixa etária (Compas, Connor-Smith, Saltzman, Thomsen, \& Wadsworth, 2001; Garcia, 2010; Sveinbjornsdottir \& Thorsteinsson, 2008).

Por estes motivos, o presente trabalho pretende centrarse nas estratégias que os adolescentes utilizam para lidar com estas situações ou contextos mais desafiantes - as chamadas estratégias de coping. Para isso, os autores propõem-se explorar, na população portuguesa, as características psicométricas de uma versão, reduzida, de uma das escala de coping mais estudadas junto de adolescentes, a Adolescent Coping Orientation for Problem Experiences (Patterson \& McCubbin, 1987), e perceber as estratégias de coping em função de variáveis sóciodemográficas como o género e a idade.

\section{O estudo do coping}

O coping tem sido definido como o conjunto de estratégias, cognitivas e comportamentais, que os indivíduos utilizam para gerir, reduzir, minimizar e lidar com as exigências internas e externas (Lewis \& Frydenberg, 2002; ZimmerGembeck \& Skinner, 2008). Desde as perspectivas fisiológicas de Selye (1956), muitas propostas têm sido apresentadas para o estudo do stress e do coping. Apesar das diversas abordagens, a maioria dos estudos tem sido influenciada pela teoria transacional ou interaccionista de coping de Lazarus (1980). Nesta perspectiva, são essenciais dois tipos de processos na resposta aos acontecimentos stressantes: a avaliação sobre as qualidades desse stressor (o potencial de ameaça); e as estratégias de coping que o indivíduo pode utilizar para lidar com esses acontecimentos stressantes. Dessa forma, os processos de avaliação e o coping são, assim, entendidos como processos transacionais, na medida em que existe uma constante interação entre a pessoa e o meio, continuamente em mudança (Folkman \& Lazarus, 1991).

Baseados nesses pressupostos, diferentes autores agruparam as estratégias de coping em categorias ou estratégias. De acordo com Compas e colaboradores (2001), poderíamos distinguir entre estratégias focadas no problema ou focadas na emoção. As focadas no problema envolvem esforços para mudar a fonte de stress, como a procura e recolha de informação, exploração de soluções possíveis ou ações que permitam alterar os stressores. Já as estratégias focadas na emoção envolvem a mudança da resposta emocional do indivíduo ao stress, o que pode envolver respostas como a procura de apoio ou a expressão das emoções. Se as estratégias focadas nos problemas parecem associar-se a maior saúde (McMahon et al., 2013; Penley, Tomaka, \& Weibe, 2002), as estratégias focadas nas emoções tendem a ser associadas a consequências mais negativas, por exemplo, consumo de álcool (Wills, Sandy, Yaeger, Cleary, \& Shinar, 2001), perturbações do humor (Miers, Rieffe, Terwogt, Cowan, \& Linden, 2007; Thompson et al., 2010), problemas de comportamento (Elgar, Arlett, \& Groves, 2003) ou mesmo comportamentos suicidários (McMahon et al., 2013).

Numa outra tipologia, Frydenberg e colaboradores (2004) distinguem entre estratégias: as focadas no problema, com o objetivo de lidar positivamente com o problema, mantendo otimismo em relação à sua resolução; referência a outros, envolve o contacto e suporte da rede social de apoio dos adolescentes, por exemplo, os pares, amigos, família, professores; e as estratégias não-produtivas, que envolvem estratégias respostas emocionais negativas ou estratégias de evitamento como por exemplo, ignorar o problema, preocupar ou auto-culpabilizar-se (Frydenberg \& Lewis, 1991, 2004). Enquanto as duas primeiras são entendidas como mais adaptativas, as estratégias emocionais têm sido associadas a efeitos mais negativos na saúde física e psicológica (e.g.: Aldao, Nolen-Hoeksema, \& Schweizer, 2010; Frydenberg \& Lewis, 1991, 2004).

Apesar destas evidências, os estudos têm sido realizados essencialmente sobre jovens e adultos, sendo mais escassa a investigação realizada junto das populações mais jovens (Frydenberg, 2014; Schwarzer \& Schwarzer, 1996; ZimmerGembeck \& Skinner, 2011). Aliás, grande parte da avaliação do coping tem sido centrada nas orientações dos adultos (e. g., Garcia, 2010) e só depois adaptada para as populações mais jovens, o que contribui para a manutenção de dúvidas e controvérsia quanto à sua conceptualização, dimensionalidade, número e tipo de estratégias mais eficazes (Boubeta, Iglesia, Ongarato, \& Liporace, 2011; Skinner, Edge, Altman, \& Sherwood, 2003). Considerando as mudanças desenvolvimentais nesta faixa etária importante para o estabelecimento de estilos de vida, importa explorar melhor estes processos junto da população adolescente (Garcia, 2010).

\section{Avaliação do coping na adolescência}

Especialmente desde a década de 90 tem-se registado um aumento progressivo na investigação no âmbito do coping na adolescência (Compas et al., 2001), embora com menor investimento quando comparado com o estudo na infância e idade adulta (Schwarzer \& Schwarzer, 1996). No entanto, a importância do seu estudo é evidente, sendo referido como um fator essencial no ajustamento dos adolescentes (Compas et al., 2001), permite-nos compreender e desenhar estratégias preventivas adequadas, localizar apoios adequados ou ajudar o adolescente a lidar adaptativamente com os desafios que se lhes colocam (Zimmer-Gembeck \& Skinner, 2008; Zimmer-Gembeck \& Skinner, 2011). Em geral, os resultados tendem a sugerir que uso predominante de formas de coping mais funcionais, é independente à cultura (Gelhaar et al., 2007). No entanto, em termos diferenciais, 
os estudos realizados junto de adolescentes têm permitido perceber um reportório mais associado à resolução de problemas nas moças, partilha com outros significativos, procura de ajuda ou manutenção de uma atitude positiva, enquanto que os rapazes tendem a usar estratégias menos eficazes, como comportamentos agressivos (Bermúdez, Teva, \& Buela-Casal, 2009; Campos, Delgado, \& Jiménez, 2012; Gelhaar et al., 2007; Rodríguez, Torres, \& Páez, 2012). No entanto, outros dados apontam no sentido oposto, referindo-se indicadores mais problemáticos de stress interpessoal e distress emocional nas moças, recorrendo preferencialmente ao suporte social e utilizam estratégias de coping maladaptativas (Boo \& Wicherts, 2009; Hampel \& Petermann, 2006).

$\mathrm{Na}$ revisão de estudos sobre o efeito da idade sobre o coping, Zimmer-Gembeck e Skinner (2011), verificam, ao longo da idade, progressivas capacidades para a implementação de estratégias de coping, refletidas numa maior autonomia e autoconfiança, planeamento das estratégias para a resolução de problemas e utilização de estratégias comportamentais e de elaboração cognitiva; e utilização de estratégias de acordo com as que se consideram mais eficazes para lidar com stressores específicos. Também em estudos longitudinais se encontra esta tendência de aumento da utilização de estratégias de procura de ajuda e resolução do problema, assim como a diminuição de estratégias de evitamento, quando perante acontecimentos vitais stressantes entre adolescentes e jovens adultos (Campos et al., 2012). Parece ainda verificar-se uma relação positiva entre estratégias produtivas e funcionalidade familiar (Esperanza, León, \& Ortega, 2012), além de pontuações mais elevadas nas estratégias de resolução de problemas e relação com os outros entre os adolescentes que frequentam escolas privadas quando comparados com escolas públicas (Bermúdez et al., 2009).

Também na relação entre o coping e o ajustamento dos adolescentes, percebe-se uma correlação entre estratégias improdutivas com o stress quotidiano, problemas emocionais e comportamentais, escolar e clínico, desajuste pessoal, e stress, depressão e ansiedade (Hampel \& Petermann, 2006; Kristensen, Schaefer, \& Busnello, 2010; Morales-Rodríguez et al., 2012); enquanto as estratégias positivas e comportamento pró-social relacionam-se com o coping centrado no problema, na relação com os outros e solução activa (Boo \& Wicherts, 2009; Morales-Rodríguez et al., 2012; Park \& Iacocca, 2014; Sontag \& Graber, 2010).

\section{0 presente estudo}

Numa revisão crítica de escalas, Sveinbjornsdottir e Thorsteinsson (2008) verificam limitações nos instrumentos existentes, seja ao nível de informação sobre o desenvolvimento das medidas, alguma confusão semântica ou dados psicométricos mais pobres. Apesar das diferenças, estes instrumentos permitem captar um conjunto de informação relevante para a compreensão destas estratégias de coping na adolescência e contribuem para a constituição de um bom quadro de referência para novos instrumentos (Burnett \& Fanshawe, 1997). O Adolescent Coping Orientation for Problem Experiences (A-COPE) (Patterson \& McCubbin, 1987) é um dos mais utilizados na avaliação do coping (Garcia, 2010), tendo sido adaptado para diversos países (e.g.: Forns et al., 2013; Halvarsson, Lunner, \& Sjödén, 2001). Aparece na literatura como um instrumento bem-estabelecido (Blount, et al., 2008), tendo sido revisto e reduzido, mais tarde por Burnett e Fanshawe (1997), para uma versão mais curta e com propriedades psicométricas ajustadas, que queremos aqui testar. Assim, considerando a escassez de estudos e de instrumentos adaptados para a população portuguesa, o presente estudo pretende explorar as propriedades psicométricas da adaptação da Escala de Estratégias de Coping na Adolescência (Burnett \& Fanshawe, 1997), analisando o coping em função de variáveis demográficas, nomeadamente o género e a idade.

\section{Método}

\section{Participantes}

A amostra do estudo é constituída por 783 adolescentes, maioritariamente do sexo feminino (61,4\%, $n=481$, para $38,6 \%$ do sexo masculino, $n=302$ ), com idades compreendidas entre os 15 e os 18 anos $(M=16,30, D P=0,931)$, que frequentavam cursos do Ensino Secundário. Da amostra $27,8 \%$ frequentava o $10^{\circ}$ ano $(n=218), 40,1 \%$ frequentava o $11^{\circ}$ ano $(n=314)$ e $32,1 \%$ frequentava o $12^{\circ}$ ano $(n=251)$.

\section{Instrumentos}

Foi utilizada a Escala de Estratégias de Coping na Adolescência (Burnett \& Fanshawe, 1997), um instrumento que resulta do refinar da escala de 54 itens Adolescent Coping Orientation for Problem Experiences (Patterson \& McCubbin, 1987). A redução de itens e eliminação de subescalas do instrumento original deu origem à Escala de Estratégias de Coping na Adolescência, com 26 itens e 10 subescalas: melhoria pessoal; focus no positivo; procura de apoio espiritual; recurso ao humor; procura de diversões; descarga emocional; uso de drogas; procura de apoio familiar; procura de apoio de outros; procura de apoio profissional. Essas subescalas foram ainda agrupadas em três fatores de segunda-ordem ou domínios superiores do coping: resolução positiva dos problemas; respostas emocionais ao problema; e procura de apoio social para responder ao problema. Foi ainda utilizado um questionário sócio-demográfico para recolher informações sobre o gênero, idade e escolaridade dos adolescentes.

\section{Procedimento}

Para a elaboração deste trabalho foram, inicialmente, contactados os autores dos instrumentos, no sentido de obter autorização para a sua adaptação para a população portuguesa e utilização neste estudo. A Escala foi submetida a uma primeira tradução pelos autores deste trabalho, e retroversão por um tradutor nativo de um país de língua inglesa, para comparação das versões pelos autores da versão original. Com o acordo destes, a versão experimental foi discutida com um grupo piloto, no sentido de avaliar a inteligibilidade dos itens. Posteriormente foi submetida a uma amostra de conveniência após obtida autorização dos Conselhos Directivos de duas es- 
colas do norte do país. Os instrumentos foram distribuídos e preenchidos pelos adolescentes que apresentaram o Termo de Consentimento Livre e Esclarecido. Aos alunos que não apresentaram o Termo assinado pelos pais ou em que estes não autorizaram a sua participação, foi proposta a realização de uma tarefa pelos professores. No início da aplicação, na sala de aula, durante o horário letivo normal, para garantir maior uniformidade na aplicação da prova, o investigador salientou o carácter voluntário da sua participação, garantido que não eram recolhidos dados pessoais e que todos os dados seriam utilizados apenas para os fins da pesquisa.

As respostas dos adolescentes foram codificadas e analisadas no programa de tratamento estatístico Statistical Package for Social Sciences, versão 15, que permitiu perceber as características psicométricas dos instrumentos. Para este estudo instrumental (Carretero-Dios \& Pérez, 2007) foram replicados os procedimentos estatísticos originais, com o recurso à análise fatorial pelo método Maximum Likelihood, utilizando o método de Rotação Oblimin com Kaiser Normalization. A consistência interna foi calculada com o recurso ao alfa de Cronbach. As diferenças de gênero foram calculadas com base no teste $t$ de Student e a associação de variáveis intervalares com teste de correlação de Pearson.

Tabela 1

Estrutura Fatorial da Escala de Estratégias de Coping na Adolescência

\section{Resultados}

\section{Validade e fidelidade}

Através do teste de esfericidade de Bartlett e da medida de Kaiser-Meyer-Olkin, foram encontrados os valores respectivamente de 4944,90 ( $p \leq 0,01)$ e 0,83 , que atestam a adequabilidade dos dados para a análise factorial. Os 26 itens foram submetidos a uma análise fatorial exploratória forçando a extração de 10 factores. Através deste procedimento, foi possível encontrar uma solução capaz de explicar $67,72 \%$ da variância. Tal como se pode verificar pela leitura da Tabela 1 , verificando-se a existência de uma boa estrutura fatorial, com a maioria dos itens a apresentar saturações elevadas (genericamente superiores a 0.50 ) nos factores a que pertencem. Excetua-se o item 6 ("Digo coisas agradáveis para os outros"), originalmente associado à dimensão apoio aos outros, apresenta valores de saturação ligeiramente mais elevada no factor 5 , relativo à subescala Humor. Este resultado pode estar associado à escrita do item que parece não diferenciar entre ambos os construtos, pelo que se optou pela sua eliminação.

\begin{tabular}{|c|c|c|c|c|c|c|c|c|c|c|c|}
\hline & & \multicolumn{10}{|c|}{ Fator } \\
\hline & & $\mathbf{1}$ & 2 & 3 & 4 & 5 & 6 & 7 & 8 & 9 & 10 \\
\hline 9 & Vou à igreja & 0,97 & & & & & & & & & \\
\hline 22 & Rezo & 0,71 & & & & & & & & & \\
\hline 12 & Esforço-me na escola ou nos projetos escolares & & 0,80 & & & & & & & & \\
\hline 11 & Tento ser mais organizado(a) e classificar as minhas prioridades & & 0,69 & & & & & & & & \\
\hline 4 & $\begin{array}{l}\text { Tento melhorar-me (e.g. manter o meu corpo em forma, } \\
\text { obter melhores notas, etc.) }\end{array}$ & & 0,67 & & & & & & & 0,42 & \\
\hline 21 & Fumo & & & 0,80 & & & & & & & \\
\hline 10 & Uso drogas (não prescritas por um médico) & & & 0,58 & & & & & & & \\
\hline 24 & Bebo álcool (e.g. cerveja, vinho, licor) & & & 0,58 & & & & & & & \\
\hline 16 & Falo com a minha mãe sobre o que me preocupa & & & & 0,76 & & & & & & \\
\hline 26 & Falo com os meus pais sobre o que o me preocupa & & & & 0,73 & & & & & & \\
\hline 20 & Faço coisas com a minha família & & 0,47 & & 0,53 & & & & & & $-0,46$ \\
\hline 8 & Brinco e mantenho um sentido de humor & & & & & $-0,81$ & & & & 0,41 & \\
\hline $\mathbf{1}$ & Tento ser engraçado e compreender o que se passou & & & & & $-0,53$ & & & & & \\
\hline 6 & Digo coisas agradáveis para os outros & & & & & 0,48 & & & 0,36 & $-0,41$ & \\
\hline 25 & Digo coisas más às pessoas & & & & & & 0,64 & & & & \\
\hline 7 & Enfureço-me e grito com as pessoas & & & & & & 0,62 & & & & \\
\hline 13 & Culpo os outros pelo que ocorre errado & & & & & & 0,56 & & & & \\
\hline 17 & $\begin{array}{l}\text { Procuro aconselhamento profissional (sem ser de um } \\
\text { professor ou de um psicólogo escolar) }\end{array}$ & & & & & & & $-0,74$ & & & \\
\hline 3 & Converso com um professor ou psicólogo escolar sobre o que me preocupa & & & & & & & $-0,65$ & & & \\
\hline 15 & Tento ajudar outras pessoas a resolver os seus problemas & & & & & & & & $-0,74$ & & \\
\hline 14 & Gasto tempo com alguém com quem me preocupo & & & & & & & & $-0,63$ & & \\
\hline 18 & Tento manter as amizades ou fazer novos amigos & & 0,44 & & & & & & $-0,51$ & 0,42 & \\
\hline 5 & Tento concentrar-me nas boas coisas da vida & & & & & & & & & 0,80 & \\
\hline 23 & Tento ver as coisas boas de uma situação difícil & & & & & & & & & 0,47 & \\
\hline 19 & Vou ao cinema & & & & & & & & & & $-0,73$ \\
\hline 2 & Vou ver lojas e compro coisas de que eu gosto & & & & & & & & & & $-0,46$ \\
\hline
\end{tabular}


Através do cálculo do alfa de Cronbach, foi encontrado um valor de alfa total de 0.77 , variando entre 0.45 na subescala procura de diversões até 0.80 na subescala procura de apoio espiritual.

\section{Correlações entre estratégias de coping}

Na tabela 2, apresenta-se a matriz de correlações entre as estratégias de coping. Estas permitem verificar uma relação estatisticamente significativa baixa ou média entre a maioria das estratégias, exceto entre a descarga emocional, procura de apoio espiritual, recurso ao humor e procura de diversões; uso de drogas, focus no positivo, procura de apoio espiritual e procura de apoio dos outros; e procura de apoio profissional, descarga emocional e uso de drogas.

Tabela 2

Correlações entre as Estratégias de Coping na Adolescência

\section{Análise fatorial de $2^{a}$ ordem}

Tal como na versão original, uma análise fatorial de segunda ordem, com o recurso à rotação maximum likehood-oblim com valor próprio superior a 1 , permitiu identificar três fatores de ordem superior com valor próprio superior a 0.40 (Tabela 3). O primeiro fator - abordar o problema de forma positiva - agrupa as subescalas procura de apoio dos outros, focus no positivo, recurso ao humor e melhoria pessoal; o segundo fator - resposta emocional - agrupa as subescalas utilizar drogas e descarga emocional; e no terceiro fator - procura de apoio social - saturam as subescalas procura de apoio de familiares, de profissionais, apoio espiritual e procura de diversões.

\begin{tabular}{|c|c|c|c|c|c|c|c|c|c|}
\hline & $\mathbf{1}$ & 2 & 3 & 4 & 5 & 6 & 7 & 8 & 9 \\
\hline 1. Melhoria pessoal & - & & & & & & & & \\
\hline 2. Focus no positivo & $0,37^{* *}$ & - & & & & & & & \\
\hline 3. Procura de apoio espiritual & $0,20^{* *}$ & $0,09^{*}$ & - & & & & & & \\
\hline 4. Recurso ao humor & $0,18^{* *}$ & $0,33^{* *}$ & $0,09^{*}$ & - & & & & & \\
\hline 5. Procura de diversões & $0,32^{* *}$ & $0,20^{* *}$ & $0,20^{* *}$ & $0,25^{* *}$ & - & & & & \\
\hline 6. Descarga emocional & $-0,21^{* *}$ & $-0,17^{* *}$ & $-0,03$ & $-0,06$ & 0,04 & - & & & \\
\hline 7. Uso de drogas & $-0,11^{* *}$ & 0,02 & 0,03 & $0,14^{* *}$ & $0,18^{* *}$ & $0,28^{* *}$ & - & & \\
\hline 8. Procura de apoio familiar & $0,43^{* *}$ & $0,30^{* *}$ & $0,33^{* *}$ & $0,14^{* *}$ & $0,32^{* *}$ & $-0,19^{* *}$ & $-0,11^{* *}$ & - & \\
\hline 9. Procura de apoio dos pares & $0,46^{* *}$ & $0,40^{* *}$ & $0,18^{* *}$ & $0,37^{* *}$ & $0,35^{* *}$ & $-0,21^{* *}$ & 0,06 & $0,34^{* *}$ & - \\
\hline 10. Procura de apoio profissional & $0,27^{* *}$ & $0,15^{* *}$ & $0,31^{* *}$ & $0,08^{*}$ & $0,23^{* *}$ & $-0,07$ & $-0,01$ & $0,42^{* *}$ & $0,17^{* *}$ \\
\hline
\end{tabular}

** Correlação significativa para o nível $0,01 .{ }^{*}$ Correlação significativa para o nível 0,05.

Tabela 3

Estudo de Dimensionalidade do Coping

\section{Fator}

$\begin{array}{lll}\text { 1. Resolução positiva do problema } & \text { 2. Resposta emocional } & \text { 3. Procura de apoio social }\end{array}$

\begin{tabular}{|c|c|c|c|}
\hline Procura de apoio dos pares & 0,75 & & 0,38 \\
\hline Focus no positivo & 0,57 & & \\
\hline Recurso ao humor & 0,55 & & \\
\hline Melhoria pessoal & 0,55 & & \\
\hline Uso de drogas & & 0,62 & \\
\hline Descarga emocional & & 0,50 & \\
\hline Procura de apoio familiar & 0,39 & & 0,71 \\
\hline Procura de apoio profissional & & & 0,58 \\
\hline Procura de apoio espiritual & & & 0,48 \\
\hline Procura de diversões & 0,46 & & 0,47 \\
\hline
\end{tabular}




\section{Coping na adolescência}

Como se apresenta na tabela 4, os adolescentes da amostra aparecem em situações de stress, valorizar a procura de apoio dos outros $(M=3,87, D P=0,68)$, estratégias de focus no positivo $(M=3,86, D P=0,80)$ e tentativa de melhoria pessoal $(M=3,64, D P=0,81)$. Pelo contrário, parecem utilizar menos estratégias o uso de drogas $(M=1,66, D P=0,80)$ ou descarga emocional $(M=1,92, D P=0,74)$.

Na exploração das estratégias de coping na adolescência em função do género, verifica-se que as moças apresentam pontuações médias mais elevadas nas estratégias de coping centradas na melhoria pessoal $[t(773)=2,97, p<0,01]$, na procura de apoio espiritual $[t(774)=2,90, p<0,01]$, de diver- sões $[t(772)=3,54, \mathrm{p}=0,003]$, de apoio familiar $[t(772)=$ $2,09, p=0,04]$ e dos pares [ $\mathrm{t}(765)=3,63, p<0,01]$. Os rapazes apresentam pontuações médias mais elevadas em estratégias de recurso ao humor $[t(768)=-3,27, p<0,01]$ e uso de drogas $[t(765)=-4,74, p<0,01]$. Em função dos fa,tores de segunda ordem, os rapazes apresentam pontuações mais elevadas nas respostas emocionais $[t(743)=-2,40, p=0,02]$ enquanto as moças apresentam pontuações mais elevadas na procura de apoio social $[t(749)=3,54, p<0,01)$.

Verificou-se ainda uma relação positiva entre idade e uso de drogas $(r=0,21, p<0,01)$ e uma relação negativa entre idade e procura de apoio da família $(r=-0,08, p=0,03)$ e apoio profissional $(r=-0,07, p=0,05)$. Na análise de segunda ordem, verifica-se uma relação negativa entre a idade e as respostas emocionais $(r=-0,16, p<0,01)$.

Tabela 4

Estatísticas Descritivas da Escala de Coping

\begin{tabular}{lrrrrrrrrrr}
\hline & \multicolumn{3}{c}{ Estatísticas descritivas } & \multicolumn{3}{c}{ Rapazes } & \multicolumn{2}{c}{ Moças } & \multicolumn{2}{c}{ Mann Whitney } \\
\cline { 2 - 10 } & Min. & Máx. & $\boldsymbol{M}$ & $\boldsymbol{D P}$ & $\boldsymbol{M}$ & $\boldsymbol{D P}$ & $\boldsymbol{M}$ & $\boldsymbol{D P}$ & $\boldsymbol{Z}$ & $\boldsymbol{p}$ \\
\hline 1. Melhoria pessoal & 3,00 & 15,00 & 10,85 & 2,37 & 10,54 & 2,38 & 11,05 & 2,35 & $-3,15$ & 0,00 \\
2. Focus no positivo & 2,00 & 10,00 & 7,66 & 1,58 & 7,70 & 1,59 & 7,64 & 1,57 & $-0,64$ & 0,52 \\
3. Procura de apoio espiritual & 2,00 & 10,00 & 4,38 & 2,31 & 4,08 & 2,23 & 4,57 & 2,34 & $-3,07$ & 0,00 \\
4. Recurso ao humor & 2,00 & 10,00 & 6,89 & 1,73 & 7,18 & 1,65 & 6,70 & 1,76 & $-3,45$ & 0,00 \\
5. Procura de diversões & 2,00 & 10,00 & 6,06 & 2,06 & 5,76 & 2,08 & 6,24 & 2,03 & $-3,39$ & 0,00 \\
6. Descarga emocional & 3,00 & 15,00 & 5,86 & 2,22 & 5,79 & 2,39 & 5,90 & 2,12 & $-1,32$ & 0,19 \\
7. Uso de drogas & 3,00 & 15,00 & 5,04 & 2,42 & 5,60 & 2,71 & 4,68 & 2,13 & $-5,00$ & 0,00 \\
8. Procura de apoio familiar & 3,00 & 15,00 & 9,36 & 2,85 & 9,08 & 3,02 & 9,54 & 2,72 & $-1,94$ & 0,05 \\
9. Procura de apoio dos pares & 4,00 & 20,00 & 15,54 & 2,66 & 15,14 & 2,90 & 15,80 & 2,47 & $-2,86$ & 0,00 \\
10. Procura de apoio profissional & 2,00 & 10,00 & 4,20 & 1,87 & 4,12 & 1,93 & 4,25 & 1,83 & $-1,20$ & 0,23 \\
\hline
\end{tabular}

\section{Discussão}

No presente estudo pretendíamos apresentar as propriedades psicométricas da adaptação da Escala de Estratégias de Coping na Adolescência (Burnett \& Fanshawe, 1997) para a população portuguesa. Os resultados encontrados permitem sustentar a manutenção da estrutura fatorial original, com a generalidade dos itens a apresentar saturações mais elevadas nos fatores esperados. Excetua-se o item 6 , que se refere à partilha de comentários agradáveis, originalmente associado à procura de apoio dos outros, mas que neste estudo apresenta valor próprio mais elevado em fatores relativos à procura de apoio de profissionais ou focus no positivo. Pelo baixo poder de diferenciação do item, deve-se equacionar a sua eliminação ou reescrita, de forma a preservar a estrutura interna da escala. Também, tal como a versão original (Burnett \& Fanshawe, 1997), a subescala com mais baixa consistência interna foi a procura de diversões, o que, eventualmente, pode estar relacionado com as diferenças entre rapazes e moças. Além disso, não devemos deixar de considerar o efeito do número de itens reduzido no valor de consistência interna.

Pela existência de um conjunto tão alargado de correlações entre fatores de primeira ordem, colocou-se a hipótese da existência de uma estrutura subjacente às estratégias avaliadas. E, de faco, uma análise fatorial exploratória de segun- da ordem permitiu encontrar uma estrutura de três factores consistentes com as versões anteriores (Burnett \& Fanshawe, 1997; Frydenberg et al., 2004), com valores de saturação superiores a 0,4 . O primeiro fator, designado de abordagem positiva do problema aparece constituído pelas subescalas procura de apoio dos outros, focus no positivo, sentido de humor e melhoria pessoal. No segundo fator, designado de resposta emocional, são agrupadas as subescalas relativas ao uso de drogas e descarga emocional. Na procura de apoio social, são agrupadas as referências a familiares, profissionais, apoio espiritual e procura de diversões. Contrariamente ao encontrado na versão original, em que a procura de diversão aparece relacionada com a abordagem positiva ao problema, resultados que permitem encontrar uma relação entre procura de diversões e procura de apoio social emergem, embora as diferenças nas cargas sejam pequenas para o primeiro fator. Percebe-se, assim, a dificuldade desta escala diferenciar entre abordagem positiva do problema ou a procura de apoio social. Apesar de alguns aspectos a confirmar em próximos estudos, estes dados sugerem o carácter multidimensional do coping (Boubeta et al., 2011; Skinner et al., 2003), e em acordo com as restantes adaptações culturais do instrumento (Forns et al., 2013; Halvarsson, Lunner, \& Sjödén, 2001).

Analisando as estratégias que os adolescentes utilizam em situações de stress verificamos uma maior utilização de procura de apoio dos outros, estratégias de focus no positi- 
vo e tentativa de melhoria pessoal. Tais dados parecem indicar uma maior tendência para a utilização de estratégias adaptativas na forma como lidam com o stress, na linha de estudos anteriores que apontam o uso mais prevalente de estratégias focalizadas nos problemas (Frydenberg, 2014; Lewis \& Frydenberg, 2002; McMahon et al., 2013; Penley, Tomaka, \& Weibe, 2002). No sentido contrário, parece ser menos utilizadas estratégias de procura como o apoio espiritual, de apoio profissional, uso de drogas ou descarga emocional. Deve ser considerado que este estudo considerou as estratégias de coping utilizadas pelos adolescentes, de forma geral, na mesma linha que estudos anteriores (Kristensen et al., 2010) mas que não são os mais utilizados, que se centram em stressores específicos.

Tal como decorre de investigação anterior, parecem existir diferenças nas estratégias de coping em função do gênero, com as moças a recorrer mais frequentemente à procura de apoio familiar, espiritual e dos outros e diversões, estratégias mais focadas na procura de apoio dos outros e melhoria pessoal. Estes resultados vão de encontro à maioria da investigação, que refere que as mulheres tendem a procurar mais o apoio social (Bermúdez et al., 2009; Campos et al., 2012; Frydenberg \& Lewis, 1991; Rodríguez et al., 2012), estratégias que podem, muitas vezes ser também associadas à dependência, em vez de revelar uma capacidade de adaptação às situações stressantes (Lewis \& Frydenberg, 2002). No entanto, percebe-se também a utilização mais frequente de estratégias positivas como a procura da melhoria pessoal, contrariamente a um conjunto de investigação que sugere que as moças tendem a utilizar estratégias de coping focadas na emoção ou evitamento da situação problemática (Boo \& Wicherts, 2009; Frydenberg \& Lewis, 1991; Hampel \& Ptermann, 2006; Rodríguez, Torres, \& Páez, 2012). Por outro lado, os rapazes da amostra utilizam mais frequentemente estratégias disfuncionais, seja pela utilização de drogas ou procura de humor. Enquanto a primeira estratégia, claramente disfuncional (Bermúdez et al., 2009; Frydenberg \& Lewis, 1991; Rodríguez et al., 2012), a segunda pode estar associada a uma resolução mais positiva da situação desafiadora (Erickson \& Feldstein, 2007; Merza et al., 2009).

No que diz respeito à relação entre as estratégias de coping e idade, verifica-se uma maior tendência para a utilização do uso de substâncias e diminuição da procura de apoio da família e de profissionais. Estes resultados parecem ir de encontro ao encontrado na literatura, que associa a diminuição do coping funcional, como as respostas emocionais, ao longo da idade (Compas et al., 2001; Frydenberg, 2004, 2014), seja pela ocorrência de adversidades ou situações de stress que o adolescente não consegue ultrapassar ou outros desafios para a utilização de estratégias adequadas (Campos et al., 2012; Zimmer-Gembeck \& Skinner, 2008). A procura de maior autonomia (Zimmer-Gembeck \& Skinner, 2011), numa etapa do desenvolvimento em que a distância com a família tende a aumentar e é maior proximidade ao grupo de pares, pode ajudar a explicar a relação negativa com o apoio da família e as experiências de consumo de drogas, tantas vezes associadas à proximidade ou pressão entre os pares (Wills et al., 2001). Ao contrário de outros estudos, encontrou-se uma relação negativa com a procura de ajuda de profissionais (Campos et al., 2012; Zimmer-Gembeck \& Skinner, 2011). Estes da- dos podem supor uma procura pela autonomia, mas podem também refletir ainda algum estereótipo negativo em relação aos profissionais ou ao tipo de serviços que podem prestar. Indicações que importará aprofundar em estudos posteriores.

\section{Conclusões}

Este estudo instrumental permitiu perceber a boa adaptação desta medida para o contexto português, contribuindo também para o melhor conhecimento do coping na adolescência e a disponibilização de um instrumento com boas propriedades para a investigação e a avaliação psicológica. Contudo, é de considerar a necessidade da continuação de estudos, para a confirmação da estrutura fatorial do modelo teórico subjacente. Sugere-se, portanto, a replicação do estudo junto de novas amostras, nomeadamente com populações clínicas ou outros grupos específicos, e com o recurso a técnicas mais finas, de equações estruturais, para maior robustez no teste de validade do instrumento (Hair, Black, Babin, Anderson, \& Tatham, 2006).

No final deste trabalho, não podem deixar de ser consideradas algumas limitações do estudo, nomeadamente o recurso a uma amostra de conveniência. Seria ainda importante a utilização de medidas de controle para favorecer a segurança em termos da validade de construto, convergente e divergente. Existindo ainda discussões acerca da estrutura e da dimensionalidade do coping (Skinner et al., 2003), importa continuar os estudos, utilizando amostras mais diversificadas e instrumentos que permitam refinar a sua validade de construto. Tendo em conta a importância do coping no ajustamento dos indivíduos, é uma área que deve manter o interesse da investigação e prática (Compas et al., 2001).

\section{Referências}

Aldao, A., Nolen-Hoeksema, S., \& Schweizer, S. (2010). Emotion-regulation strategies across psychopathology: A meta-analytic review. Clinical Psychology Review, 30(2), 217-237. doi:10.1016/j.cpr.2009.11.004

Arbour-Nicitopouos, K. P., Faulkner, G. E., \& Irving, H. M. (2012). Multiple health-risk behaviour and psychological distress in adolescence. Journal of the Canadian Academy of Child and Adolescent Psychiatry, 21(3), 171-178. Recuperado de http://www.ncbi.nlm.nih.gov/pmc/articles/ PMC3413466/

Bermúdez, M., Teva, I., \& Buela-Casal, G. (2009). Influencia de variables sociodemográficas sobre los estilos de afrontamiento, el estrés social y la búsqueda de sensaciones sexuales en adolescentes. Psicothema, 21(2), 220226. Recuperado http://www.redalyc.org/articulo.oa?id=72711654008

Blount, R., Simons, L., Devine, K., Jaaniste, T., Cohen, L., Chambers, C., \& Hayutin, L. G. (2008). Evidence-based assessment of coping and stress in pediatric psychology. Journal of Pediatric Psychology, 33(9), 1021-1045. doi: 10.1093/jpepsy/jsm071

Boo, G. M., \& Wicherts, J. M. (2009). Assessing cognitive and behavioral coping strategies in children. Cognitive Therapy \& Research, 33(1), 1-20. doi: 10.1007/s10608-007-9135-0

Boubeta, A. R., Iglesia, G., Ongarato, P., \& Liporace, M. (2011). Dimensionalidad del inventario de afrontamiento para adolescentes y universitários. Psicothema, 23(3), 464-474. Recuperado de http://www.psicothema. com/psicothema.asp?id=3911 
Burnett, P. C., \& Fanshawe, J. P. (1997). Measuring school-related stressors in adolescents. Journal of Youth and Adolescence, 26(4), 415-428. doi: 10.1023/A:1024529321194

Campos, R. M., Delgado, A. O., \& Jiménez, A. P. (2012). Acontecimientos vitales estresantes, estilo de afrontamiento y ajuste adolescente: un análisis longitudinal de los efectos de moderación. Revista Latinoamericana de Psicología, 44(2), 39-53. doi: 10.14349/rlp.v44i2.1030

Carretero-Dios, H., \& Pérez, C. (2007). Standards for the development and review of instrumental studies: Considerations about test selection in psychological research. International Journal of Clinical and Health Psychology, 7(3), 863-882. Recuperado http://www.aepc.es/ijchp/carretero_es.pdf

Casey, B. J., Jones, R. M., Levita, L., Libby, V., Pattwell, S., Rubery, E., ... Somerville, L. H. (2010). The storm and stress of adolescence: Insights from human imaging and mouse genetics. Developmental Psychobiology, 2(3), 225-235. doi: 10.1002/dev.20447

Compas, B. E., Connor-Smith, J. K., Saltzman, H., Thomsen, A. H., \& Wadsworth, M. E. (2001). Coping with stress during childhood and adolescence: Problems, progress, and potential in theory and research. Psychological Bulletin, 127(1), 87-127. doi: 10.1037//0033-2909.127.1.87

Elgar, F. J., Arlett, C., \& Groves, R. (2003). Stress, coping, and behavioural problems among rural and urban adolescents. Journal of Adolescence, 26 , 574-585. doi: 10.1016/S0140-1971(03)00057-5

Erickson, S. J., \& Feldstein, S. W. (2007). Adolescent humor and its relationship to coping, defense strategies, psychological distress, and well-being. Child Psychiatry and Human Development, 37(3), 255-271. doi: 10.1007/s10578-006-0034-5

Esperanza, Z. P., León, S. F., \& Ortega, L. Y. (2012). Relación entre funcionalidad familiar y las estrategias de afrontamiento utilizadas por adolescentes embarazadas. Revista Ciencia y Cuidado, 9(2), 9-16. Recuperado de http://dialnet.unirioja.es/servlet/articulo?codigo $=4114484$

Fanshawe, B. P., \& Burnett, P. C. (1991). Assessing school-related stressors and coping. Mechanisms in adolescents. British Journal of Educational Psychology, 61, 92-98. doi: 10.1111/j.2044-8279.1991.tb00964.x

Folkman, S., \& Lazarus, R. S. (1991). Coping and emotion. In A. Monat \& R. S. Lazarus (Orgs.), Stress and coping: An anthology (pp. 207-227). Nova Iorque: Columbia University Press.

Forns, M., Kirchner, T., Peró, M., Pont, E., Abad, J., Soler, L., \& Paretilla, C., (2013). Factor structure of the adolescent coping orientation for problem experiences in Spanish adolescents. Psychological Reports: Measures, \& Statistics, 112(3), 845-871. doi: 10.2466/03.20.PR0.112.3.845-871

Frydenberg, E. (2004). Coping competencies: What to teach and when. Theory Into Practice, 43(1), 14-22. doi: 10.1207/s15430421tip4301_3

Frydenberg, E. (2014). Coping research: Historical background, links with emotion, and new research directions on adaptive processes. Australian Journal of Psychology, 66(2), 82-92. doi: 10.1111/ajpy.12051

Frydenberg, E., \& Lewis, R (1991). Adolescent coping: The different ways in which boys and girls cope. Journal of Adolescence, 14, 119-133. doi: 10.1016/0140-1971(91)90025-M

Frydenberg, E., \& Lewis, R. (2004). Adolescents least able to cope: How do they respond to their stresses? British Journal of Guidance and Counselling, 32, 25-37. doi: 10.1080/03069880310001648094

Frydenberg, E., Lewis, R., Bugalski, K., Cotta, A., McCarthy, C., Luscombe-Smith, N., \& Poole, C. (2004). Prevention is better than cure: Coping skills training for adolescents at school. Educational Psychology in Practice, 20(2), 117-134. doi: 10.1080/02667360410001691053

Garcia, C. (2010). Conceptualization and measurement of coping during adolescence: A review of the literature. Journal of Nursing Scholarship, 42(2), 166-185. doi: 10.1111/j.1547-5069.2009.01327.x

Gelhaar, T., Seiffge-Krenke, I., Borge, A., Cicognani, E., Cunha, M., Loncaric, D., ... Metze, C. W. (2007). Adolescent coping with everyday stressors: A seven-nation study of youth from central, eastern, southern, and northern Europe. European Journal of Developmental Psychology, 4(2),
129-156. doi: 10.1080/17405620600831564

Gestsdottir, G., \& Lerner, R. M. (2008). Positive development in adolescence: The development and role of intentional self-regulation. Human Development, 51(3), 202-224. doi: 10.1159/000135757

Hair, J. F., Black, W. C., Babin, B. J., Anderson, R. E., \& Tatham, R. L. (2006) Multivariate data analysis. Londres: Pearson.

Halvarsson, K., Lunner, K., \& Sjödén, P-O. (2001). Development of a Swedish version of the adolescent coping orientation for problem experiences (A-Cope). Scandinavian Journal of Psychology, 42(5), 383-388. doi: 10.1111/1467-9450.00250

Hampel, P., \& Petermann, F. (2006). Perceived stress, coping and adjustment in adolescents. Journal of Adolescent Health, 38(4), 409-415. doi: 10.1016/j.jadohealth.2005.02.014

Kenny, R., Dooley, B., \& Fitzgerald, A. (2013). Interpersonal relationships and emotional distress in adolescence. Journal of Adolescence, 36(2), 351 360. doi: 10.1016/j.adolescence.2012.12.005

Kristensen, C. H., Schaefer, L. S., \& Busnello, F. B. (2010). Estratégias de coping e sintomas de stress na adolescência. Estudos de Psicologia, 28(1), 21-30. doi: 10.1590/S0103-166X2010000100003

Lazarus, R. S. (1980). The stress and coping paradigm. In C. Eisendorfer, D. Cohen, A. Kleinman., \& P. Maxim (Orgs.), Theoretical basis for psychopathology (pp. 287-322). Nova Iorque: Spectrum.

Lazarus, R. S., \& Launier, R. (1978). Stress-related transactions between person and environment. In L. A. Pervin \& M. Lewis (Orgs), Perspectives in Interactional Psychology (pp. 287-327). Nova Iorque: Plenum.

Lewis, R., \& Frydenberg, E. (2002). Concomitants of failure to cope: What we should teach adolescents about coping. British Journal of Educational Psychology, 72(3), 419-431. doi: 10.1348/000709902320634483

McMahon, E. M., Corcoran, P., McAuliffe, C., Keeley, H., Perry, I. J., \& Arensman, E. (2013). Mediating effects of coping style on associations between mental health factors and self-harm among adolescents. Crisis, 34(4), 242-250. doi: 10.1027/0227-5910/a000188

Merza, E. L., Malcarneab, V. L., Hansdottirac, I., Furstd, D. E., Clementsb, P. J., \& Weismane, M. H. (2009). A longitudinal analysis of humor coping and quality of life in systemic sclerosis. Psychology, Health, \& Medicine, 14(5), 553-566. doi: 10.1080/13548500903111798

Miers, A. C., Rieffe, C., Terwogt, M. M., Cowan, R., \& Linden, W. (2007). The relation between anger coping strategies, anger mood and somatic complaints in children and adolescents. Journal of Abnormal Child Psychology, 35, 653-664. doi: 10.1007/s10802-007-9120-9

Morales-Rodríguez, F. M., Trianes, M. V., Blanca, M. J., Miranda, J., Escobar M., \& Fernández-Baena, F. J. (2012). Escala de Afrontamiento para Niños (EAN): propiedades psicométricas. Anales de Psicología, 28(2), 475483. doi: 10.6018/analesps.28.2.136221

Park, C. L., \& Iacocca, M. O. (2014). A stress and coping perspective on health behaviors: theoretical and methodological considerations. Anx iety, Stress, \& Coping: An International Journal, 27(2), 123-137. doi: 10.1080/10615806.2013.860969

Patterson, A. C., \& McCubbin, H. I. (1987). Adolescent coping style and behaviours: Conceptualisation and measurement. Journal of Adolescence, 1, 163-186. doi: 10.1016/S0140-1971(87)80086-6

Penley, J. A., Tomaka, J., \& Weibe, J. S. (2002). The association of coping to physical and psychological health outcomes: A meta-analytic review. Journal of Behavioral Medicine, 25(6), 551-603. doi: 10.1023/A:1020641400589

Rodríguez, F. M., Torres, M. V., \& Páez, J. M. (2012). Gender and age differences in how children cope with daily stress. Electronic Journal of Research in Educational Psychology, 10(1), 95-110.

Schwarzer, R., \& Schwarzer, C. (1996). Acritical survey of coping instruments In N. M. Zeidner \& N. S. Endler (Orgs.), Handbook of coping: Theory, research, and applications (pp. 107-132). Nova Iorque: Wiley.

Seiffge-Krenke, I., Weidemann, S., Fentner, S., Aegenheister, N., \& Poeblau, M. (2001). Coping with school-related stress and family stress in healthy 
and clinically referred adolescents. European Psychologist, 6(2), 123-132. doi: $10.1027 / / 1016-9040.6 .2 .123$

Selye, H. (1956). The stress of life. Nova Iorque: McGraw-Hill.

Skinner, E. A., \& Zimmer-Gembeck, M. J. (2007). The development of coping. Annual Review of Psychology, 58, 119-144. doi:10.1146/annurev. psych.58.110405.085705

Skinner, E. A., Edge, K., Altman, J., \& Sherwood, H. (2003). Searching for the structure of coping: A review and critique of category systems for classifying ways of coping. Psychological Bulletin, 129(2), 216-269. doi:10.1037/0033-2909.129.2.216

Sontag, L. M., \& Graber, J. A. (2010). Coping with perceived peer stress: Gender-specific and common pathways to symptoms of psychopathology. Developmental Psychology, 46(6), 1605-1620. doi: 10.1037/a0020617

Sveinbjornsdottir, S., \& Thorsteinsson, E. B. (2008). Adolescent coping scales: A critical psychometric review. Scandinavian Journal of Psychology, 49, 533-548. doi: 10.1111/j.1467-9450.2008.00669.x

Thompson, R. J., Mata, J., Jaeggi, S. M., Buschkuehl, M., Jonides, J., \& Gotlib, I. H. (2010). Maladaptive coping, adaptive coping, and depressive symptoms: Variations across age and depressive State. Behaviour Research and Therapy, 48(6), 459-466. doi: 10.1016/j.brat.2010.01.007

Wills, T. A., Sandy, J. M., Yaeger, A. M., Cleary, S. D., \& Shinar, O. (2001). Coping dimensions, life stress, and adolescent substance use: A latent growth analysis. Journal of Abnormal Psychology, 11, 309-323. doi: 10.1037/0021-843X.110.2.309

Zimmer-Gembeck, M. J., \& Skinner, E. A. (2008). Adolescents' coping with stress: development and diversity. Prevention Researcher, 15, 3-7.

Zimmer-Gembeck, M. J., \& Skinner, E. A. (2011). The development of coping across childhood and adolescence: An integrative review and critique of research. International Journal of Behavioral Development, 35(1), 1-17. doi: $10.1177 / 0165025410384923$ 
Paulo César Azevedo Dias, Doutor em Psicologia da Saúde, pela Universidade Miguel Hernández de Elche (UMH), Espanha, é coordenador do curso de Psicologia, na Faculdade de Filosofia da Universidade Católica Portuguesa (UCP). Endereço para correspondência: Praça da Faculdade, 1, 4710-297 Braga, Portugal. Telefone: (+351) 253208076 e fax: (+351) 253213 940. E-mail: pcdias@braga.ucp.pt

Jose A. Garcia del Castillo Rodriguez, Doutor em Psicologia, pela Universidade de Murcia (UM), é diretor do Instituto de Investigación de Drogodependencias (INID), catedrático de E.U. de Psicologia Social e professor do departamento de Psicologia da Saúde da Universidade Miguel Hernández de Elche (UMH), Espanha.

E-mail: jagr@umh.es

Carmen López-Sánchez, Doutora em Psicologia, pela Universidade Miguel Hernández de Elche (UMH), Espanha, é professora Titular de Universidade na área de Psicologia Social, no departamento de Comunicação e Psicologia Social da Faculdade de Ciencias Económicas e Empresarais da Universidade de Alicante (UA).

E-mail: mc.lopez@ua.es 